\title{
PREKÄRE ARBEITSVERHÄLTNISSE IM BIBLIOTHEKSWESEN
}

von Ute Wödl

Zusammenfassung: In diesem Beitrag werden die Ergebnisse der Tätigkeit der VÖB-Arbeitsgruppe „Prekäre Arbeitsverhältnisse im Bibliothekswesen" von 20162018 präsentiert. Hervorgegangen aus einer Bottom-Up-Initiative, welche in einer Podiums- und Publikumsdiskussion beim Österreichischen Bibliothekartag in Wien 2015 mündete, wurde vom VÖB-Präsidium eine offene Arbeitsgruppe ins Leben gerufen. Der Schwerpunkt der AG-Arbeit lag auf der Ausarbeitung einer umfassenden Erhebung zur Arbeitssituation bei Bibliothekarlnnen in Österreich. Die Ergebnisse der von Gallup durchgeführten Online-Umfrage, an der sich mehr als 600 Kolleginnen und Kollegen beteiligten, werden in Auszügen vorgestellt und kommentiert. Erste Überlegungen zu möglichen Maßnahmen, um atypische/als prekär erlebte Arbeitsverhältnisse zu verbessern, bilden den Abschluss der Tätigkeit der Arbeitsgruppe.

Schlüsselwörter: Beschäftigungsverhältnisse; atypische Beschäftigung; prekäre Beschäftigung; Berufseinstieg; Arbeitsmarktperspektiven; Arbeitsanforderungen; Berufszufriedenheit

\section{PRECARIOUS EMPLOYMENT IN THE LIBRARY SECTOR}

Abstract: This article presents the results the VÖB team working on "precarious employment in the library sector" has gathered from 2016-2018. Since a bottom-up-initiative resulted in a panel discussion at the Austrian Library Congress 2015 in Vienna, the VÖB executive committee decided to establish a working group on this subject. The activities focused on developing a survey in order to get information about atypical/precarious employment in the library sector. The survey was conducted by Gallup and more than 600 colleagues participated. The results are to a great extent presented and commented on in this article. Finally observations on possible measures to be taken to improve atypical/precarious employment are made.

Keywords: employment relationships; atypical employment; precarious employment; young professionals; employment prospects; job requirements; vocational satisfaction

DOI: https://doi.org/10.31263/voebm.v71i3-4.2164

(c) Ute Wödl



Dieses Werk ist lizenziert unter einer

Creative-Commons-Lizenz Namensnennung 4.0 International 


\section{Inhalt}

1. Die Vorgeschichte

2. Die Tätigkeiten der Arbeitsgruppe 2016/2017

3. Die Umfrage - ausgewählte Ergebnisse

4. Zusammenfassung - erste Schlussfolgerungen

\section{Die Vorgeschichte}

Individuelle Unzufriedenheiten mit der jeweils aktuellen beruflichen Situation gab es aus den unterschiedlichsten Gründen wohl immer. Mit Inkrafttreten des - als Folge des UG 2002 entstandenen - neuen Kollektivvertrags für die Arbeitnehmerlnnen der Universitäten $2009^{1}$ nahmen diese Unzufriedenheiten laufend zu. Dies lag u.a. darin begründet, dass seit Einführung des Universitätslehrgangs Mitte der 2000er Jahre zwar die Möglichkeit bestand, auch ohne vorherige Anstellung im Bibliothekswesen eine entsprechende Ausbildung zu absolvieren, diese Ausbildungskosten aber von den Auszubildenden selbst zu tragen waren. Darüber hinaus lagen die Einstiegsgehälter im neuen Kollektivvertrag kaum über, oft sogar unter den Einstiegsgehältern der alten Gehaltsschemata, unbefristete Stellen und/oder Vollzeitstellen waren immer schwerer zu erlangen und der Konkurrenzdruck wuchs auf Grund der hohen Zahl an neu ausgebildeten Bibliotheks- und InformationsexpertInnen. Zudem hatte die Österreichische Nationalbibliothek als eine der größten ArbeitgeberInnen für wissenschaftliche Bibliothekarlnnen überhaupt keinen Kollektivvertrag, das Gehaltsschema wurde und wird auch nicht transparent gemacht. Allerdings ließ sich aus den Gehaltsangaben bei Stellenausschreibungen erkennen, dass die Bezahlung an der ÖNB noch unter der ohnedies keineswegs üppigen Abgeltung für MitarbeiterInnen an Universitätsbibliotheken lag.

Ausgelöst durch eine Stellenausschreibung der ÖNB, welche im Frühjahr 2015 über den VÖBBlog sowie die VÖB-Mailingliste bekanntgemacht wurde - für eine inhaltlich anspruchsvolle Stelle mit benötigten bibliothekarischen Qualifikationen wurde ca. EUR 1.650,- brutto/Monat bei Vollzeit als Mindestgehalt angegeben -, entzündete sich via Mailingliste eine heftige Diskussion um Arbeitsbedingungen und Gehaltsstrukturen im österreichischen Bibliothekswesen.

Die VÖB trug dem ebenfalls im Zuge dieses Mailwechsels geäußerten Wunsch, diesem Themenkomplex im Rahmen des nächsten Bibliothekartags 2015 in Wien Raum zu geben, mit einer Podiumsdiskussion Rech- 
nung. Am 17.09.2015 leitete Monika Bargmann die Podiumsdiskussion mit dem Titel „Ich bin so frei - prekäre Arbeitsverhältnisse im Bibliothekswesen", bei der Beate Neunteufel-Zechner, Nikolaus Hamann und Werner Schlacher unterschiedliche Aspekte zu diesem Problem darlegten und auch die zahlreich anwesenden Zuhörerlnnen lebhaft mitdiskutierten - das Thema hatte ganz offensichtlich einen Nerv getroffen.

Daher beschloss das für die Funktionsperiode 2015-2017 neu gewählte VÖB-Präsidium unter Präsident Werner Schlacher die Einsetzung einer zeitlich befristeten offenen Arbeitsgruppe. Sowohl betroffene als auch allgemein an dem Thema interessierte KollegInnen sollten die Möglichkeit erhalten, sich fundiert über einen längeren Zeitraum mit dem Problem zu befassen und konkrete Maßnahmen und Änderungsvorschläge zu erarbeiten. Am 23.09.2015 wurde via VÖBBlog und VÖB-Mailingliste in einem „Call for Action“ zur Mitarbeit an dieser Arbeitsgruppe eingeladen.

Diesem Aufruf folgten 12 KollegInnen aus ganz Österreich, die sich für die Gesamtdauer des Bestandes der Arbeitsgruppe aktiv beteiligten sowie 3 KollegInnen, die sich punktuell einbrachten.

\section{Die Tätigkeiten der Arbeitsgruppe 2016/2017}

Am 13.01.2016 fand an der TU Wien/ Hauptbibliothek die konstituierende Sitzung statt. Diese erste Sitzung diente vor allem dem Austausch darüber, welchen Zugang/ welche Erfahrungen die einzelnen Teilnehmerlnnen bezüglich des Themas prekäre Arbeitssituation haben. Es wurden mögliche Ursachen und Auswirkungen der wahrgenommenen Entwicklung - am Arbeitsmarkt im Allgemeinen und im Bibliothekswesen im Speziellen - diskutiert. Weiters wurde eine Sammlung an Themenfeldern, mit denen sich die AG während der nächsten Sitzungen befassen sollte, erstellt.

Bis zum nächsten Treffen am 17.06.2016 befassten sich alle AG-Mitglieder mit den unterschiedlichen Definitionen zu atypischen und prekären Arbeitsverhältnissen, außerdem sammelten und sichteten alle diverse Statistiken zu dem Thema. Bei der Sitzung selbst wurde dann u.a. eine Priorisierung der beim ersten Treffen gesammelten Themenfelder vorgenommen, da absehbar war, dass nicht alle Bereiche abgearbeitet werden können. Dabei einigte man sich darauf, dass das Hauptaugenmerk auf der Durchführung einer statistischen Erhebung zum bibliothekarischen Arbeitsmarkt liegen soll, mit folgenden Zielsetzungen:

- Atypische Beschäftigung quantifizierbar machen durch Fragen nach Geringfügigkeit, Teilzeit, Befristung... 
- Gefahren der Prekarisierung erkennen durch Fragen nach Einkommen, Möglichkeiten der Teilnahme an Weiterbildung, Integration in betriebliche Abläufe und Informationsflüsse, Berücksichtigung bei Gratifikationen, berufliche Perspektive, Zufriedenheit mit der Tätigkeit...

- Mögliche Ansatzpunkte für Maßnahmen erkennen durch Fragen zur Ausbildung, zum Berufseinstieg, Differenz tatsächliche und Wunscharbeitszeit, Unterschied zwischen Arbeitszeit It. Vertrag und tatsächlich geleisteten Arbeitsstunden, Wünsche an die berufliche Zukunft...

Konsequenterweise beschäftigte sich die im Rahmen der ODOK am 26.09.2016 in Eisenstadt stattfindende dritte Sitzung daher ausschließlich mit den notwendigen Vorbereitungen zur Durchführung der Umfrage. Es wurde eine Liste erstellt, welche allgemeinen statistischen Daten abgefragt werden sollen, um nicht nur den Kernbereich prekäre Arbeitssituation, sondern auch damit in Zusammenhang stehende Bereiche wie Ausbildung, Berufsbild, Karriereverlauf und berufliche Zukunftsvorstellung zumindest in Teilen abbilden zu können. Zu den einzelnen Themenbereichen wurden bereits konkrete Fragestellungen formuliert. Weiters wurden mögliche Verteilungswege der Umfrage erörtert, um eine möglichst breite Streuung sowohl im wissenschaftlichen als auch im öffentlichen Bibliothekswesen zu gewährleisten, sowie das weitere Vorgehen im Hinblick auf die Beauftragung eines Instituts mit der professionellen Durchführung und Auswertung festgelegt.

Das Präsidium setzte den maximalen Betrag, den die VÖB leisten konnte, mit EUR 15.000,- fest, zudem erklärte sich die AK Wien bereit, die Umfrage mit einem Betrag von EUR 2.000,- zu unterstützen. Nun wurden 4 einschlägig bekannte Markt- und Meinungsforschungsinstitute (IFES, GALLUP, SORA und OGM) angeschrieben und um Angebotslegung ersucht. Nach Sichtung und umfassender Bewertung erhielt GALLUP auf Grund des inhaltlich umfassendsten und konkretesten, sowie auch des besten Preis-Leistungs-Angebots den Zuschlag.

In den folgenden Wochen wurde mit der zuständigen GALLUP-Mitarbeiterin Gabriele Reithner intensiv an der Ausarbeitung der Umfrage gearbeitet, bevor 10 ausgewählte BibliothekskollegInnen - die sehr heterogen zusammengesetzte Gruppe repräsentiere unterschiedliche Geschlechter, Dienstalter, Arbeitsverträge, Hierarchiestufen, Dienstgeber etc. - im Rahmen von Pretests die gestellten Fragen und vorgegebenen Antwortmöglichkeiten kritisch auf Verständlichkeit, Vollständigkeit und Nachvollziehbarkeit prüften. Auf Basis der bei den Pretests erhaltenen Rückmeldungen und Testauswer- 
tungen wurden sowohl die Fragen als auch die gewünschten Quer-Auswertungen endgültig festgelegt, bevor die Umfrage Anfang Mai 2017 umfassend über diverse Mailinglisten und Social-Media-Kanäle verteilt wurde.

Im Zeitraum 4.-22.05.2017 nahmen sich 616 Kolleginnen und Kollegen die benötigten 15-20 Minuten Zeit, um neben einigen statistisch notwendigen Angaben 47 Fragen zu ihrer Ausbildung, ihrer bisherigen Laufbahn im Bibliothekswesen, ihrer aktuellen beruflichen Situation sowie ihren beruflichen Zukunftsvorstellungen zu beantworten und uns in Freitextfeldern darüber hinaus umfassend weitere relevante und bei der Auswertung zu beachtenden Informationen zur konkreten Berufssituation zu geben.

Eine erste Präsentation der Ergebnisse durch Günter Haunlieb/GALLUP erfolgte am 19.06.2017 im Rahmen der nächsten Sitzung für die Mitglieder der AG sowie für interessierte ZuhörerInnen aus dem VÖB-Präsidium und -Vorstand. Dabei wurden nicht nur die konkreten Auswertungen zu jeder einzelnen Frage detailliert dargestellt, sondern es wurden bereits auf einige hervorstechende Werte hingewiesen und erste Vergleiche zur allgemeinen Situation am Arbeitsmarkt angestellt. Die Ergebnisse wurden anschließend diskutiert, mögliche Schwerpunktsetzungen im Hinblick auf die Präsentation beim Bibliothekartag im September wurden herausgearbeitet.

Schließlich wurde am 14.09.2017 im Rahmen einer Podiums- und Publikumsdiskussion im Themenblock „Arbeitsverhältnisse im Bibliothekswesen - wie prekär sind sie?" zuerst die bisherige Tätigkeit der AG präsentiert, also z.B. die Definition der Begriffe atypisch und prekär, Statistiken zum Medianeinkommen und zur definierten Prekariatsgrenze sowie zu Normal- und atypischen Beschäftigungsverhältnissen. Danach wurden die Ergebnisse der Umfrage sowie erste daraus gewonnene Erkenntnisse präsentiert und diskutiert.

\section{Die Umfrage - ausgewählte Ergebnisse ${ }^{2}$}

\subsection{TeilnehmerInnen}

Die Struktur der Teilnehmenden zeigt mit etwa 2/3 weiblichen Befragten auch die Struktur des Bibliothekswesens - nach wie vor wird der Beruf in erster Linie als Frauenberuf betrachtet. Bemerkenswert ist auch die Tatsache, dass auch zwei Drittel der Befragten über einen Universitätsabschluss verfügen, da die Zahl der Stellen, die ein abgeschlossenes Studium voraussetzen und entsprechend eingestuft sind, kontinuierlich zurückgeht. 


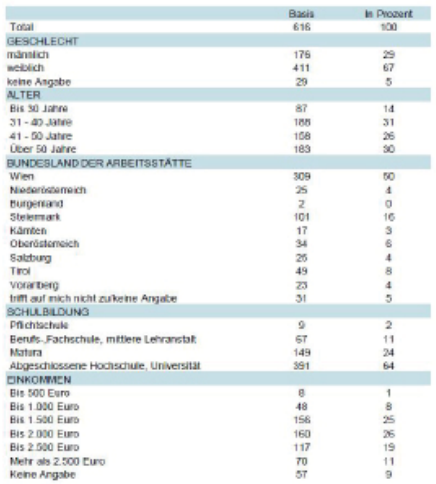

\subsection{Ausbildung}



Knapp ein Drittel der Rückmeldungen erfolgte von KollegInnen, die ihre Ausbildung erst in den vergangenen vier Jahren abschlossen, mehr als die Hälfte der Teilnehmerlnnen schlossen ihre Ausbildung in den vergangenen zehn Jahren $a b$ - sie alle absolvierten bereits den Universitätslehrgang, mussten meist 
selbst für die Finanzierung ihrer Ausbildung Sorge tragen, hatten häufig zu Beginn der Ausbildung noch wenig bis keine Erfahrung im Bibliothekswesen und sind tendenziell stärker von atypischen Arbeitsverhältnissen betroffen.

\subsection{Die bisherige Laufbahn im BI-Wesen}

3.3.1. Beschäftigungsdauer und Beschäftigungskontinuität im BI-Wesen

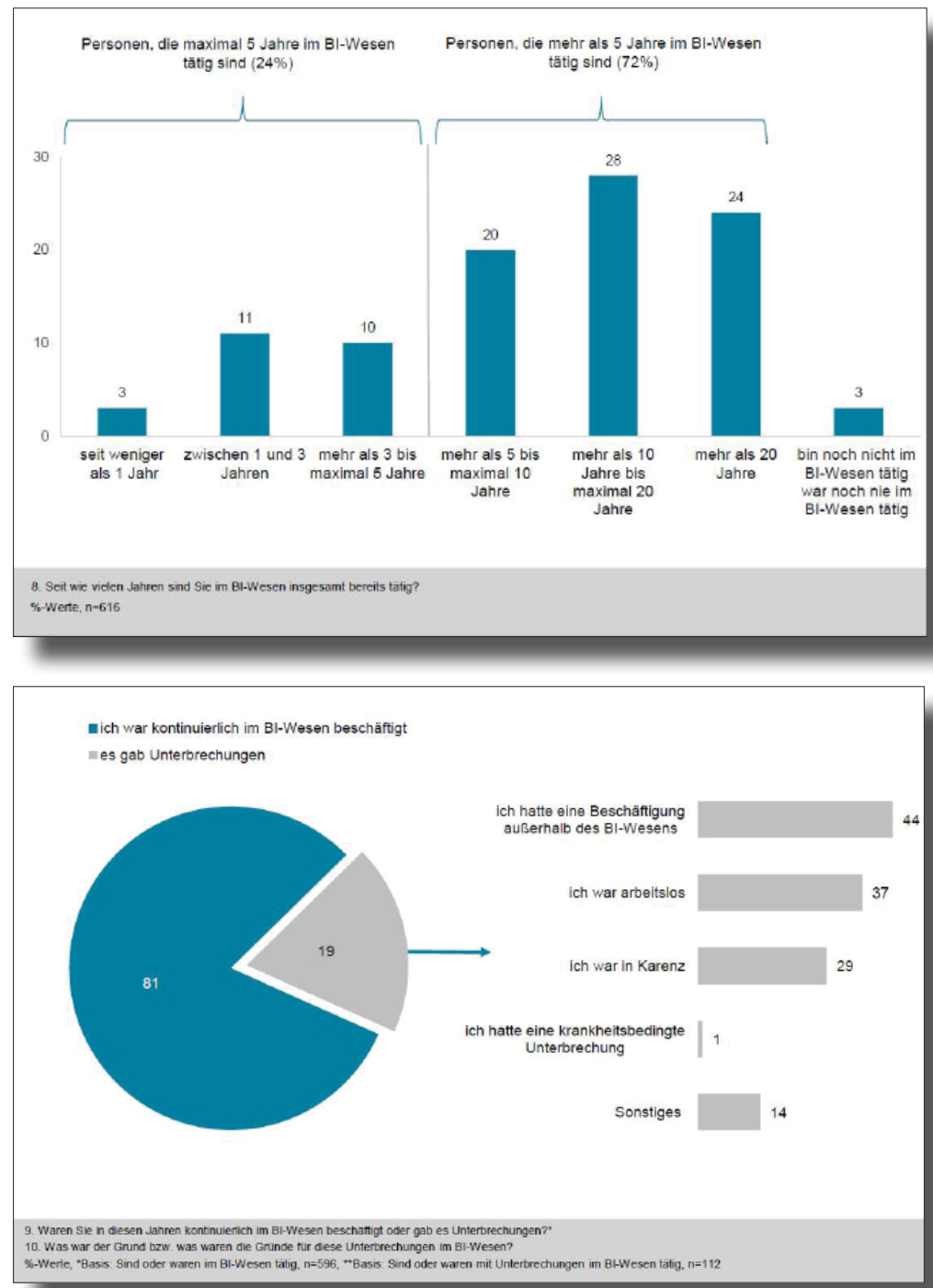


Etwa $20 \%$ der KollegInnen waren/sind nicht kontinuierlich im Bibliotheksbereich beschäftigt, wobei nicht hinterfragt wurde, ob Beschäftigungen in anderen Bereichen freiwillig angenommen wurden oder zur Überbrückung zwischen bibliothekarischen Tätigkeiten dienten. Grundsätzlich ist zu konstatieren, dass auf Grund des sich rasch ändernden beruflichen Umfelds mit jeder beruflichen Unterbrechung, vor allem wenn sie von längerer Dauer ist, auch der Wiedereinstieg erschwert wird.

\subsubsection{Dienstgeber und Arbeitsverträge}

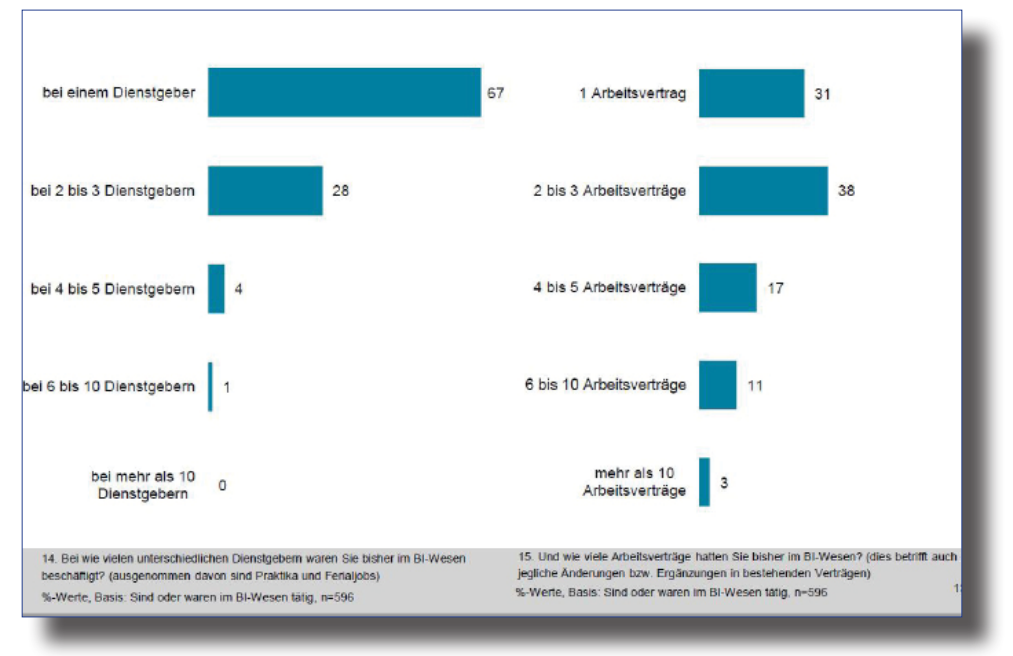

Die hohe Zahl an KollegInnen, die in ihrem Berufsleben nie oder nur äußerst selten ihren Dienstgeber wechselten, zeigt, dass Bibliothekarlnnen über eine ausgeprägte Loyalität ihrem Dienstgeber gegenüber verfügen - ein Umstand, der gerade in einem wissensbasierten Beruf für Dienstgeber große Vorteile mit sich bringt. Allerdings legt der deutliche Unterschied zwischen der Anzahl an Dienstgebern und Arbeitsverträgen nahe, dass sehr viele KollegInnen im Lauf ihres Berufslebens zuerst nur befristete Verträge erhielten.

\subsection{Die aktuelle Arbeitssituation}

\subsubsection{Vertragsverhältnis}

Diese Vermutung wird gestützt durch die Tatsache, dass fast ein Viertel derjenigen, die ihre Ausbildung erst in den vergangenen vier Jahren abschlossen, derzeit nur über einen befristeten Dienstvertrag verfügen. 


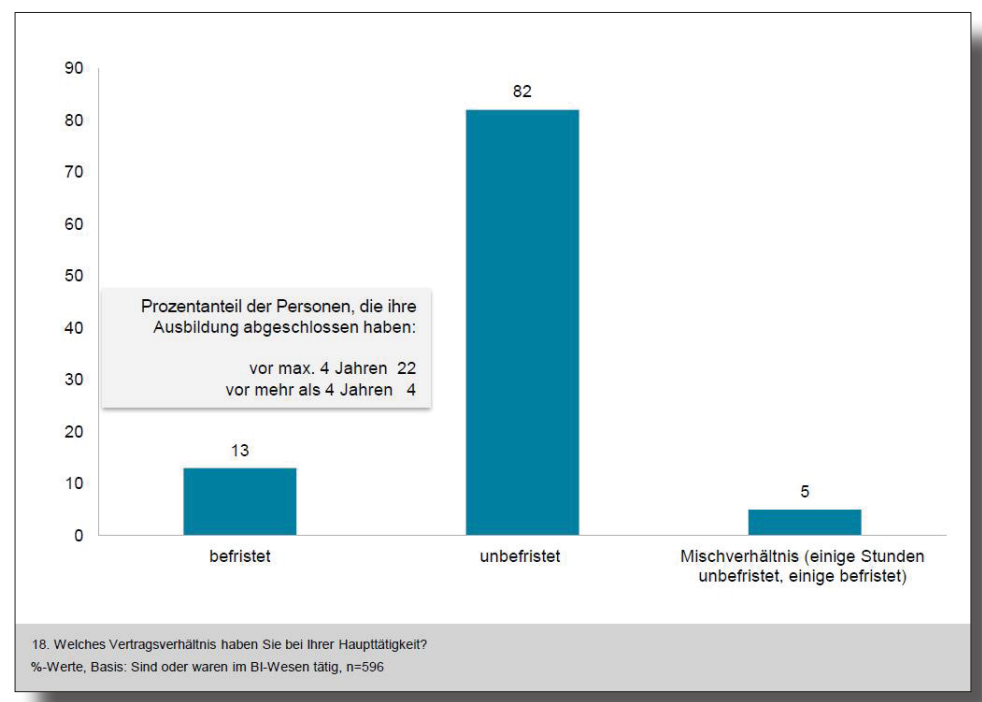

\subsubsection{Dauer der Befristung}

Die Dauer der Befristung - bei mehr als $60 \%$ der Befragten liegt sie zwischen 6 Monaten und 2 Jahren - erlaubt in den meisten Fällen eine gründliche Einarbeitung in ein Thema, ein Zusammenwachsen mit einem Team, ist aber selten lang genug, um für das berufliche und außerberufliche Leben längerfristige Planungssicherheit zu gewährleisten.

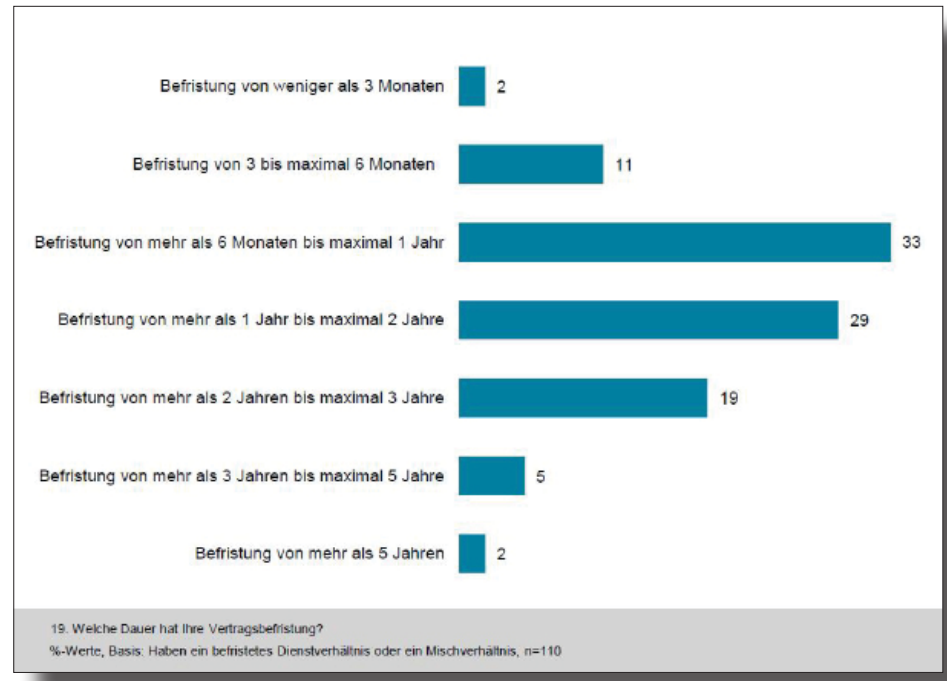




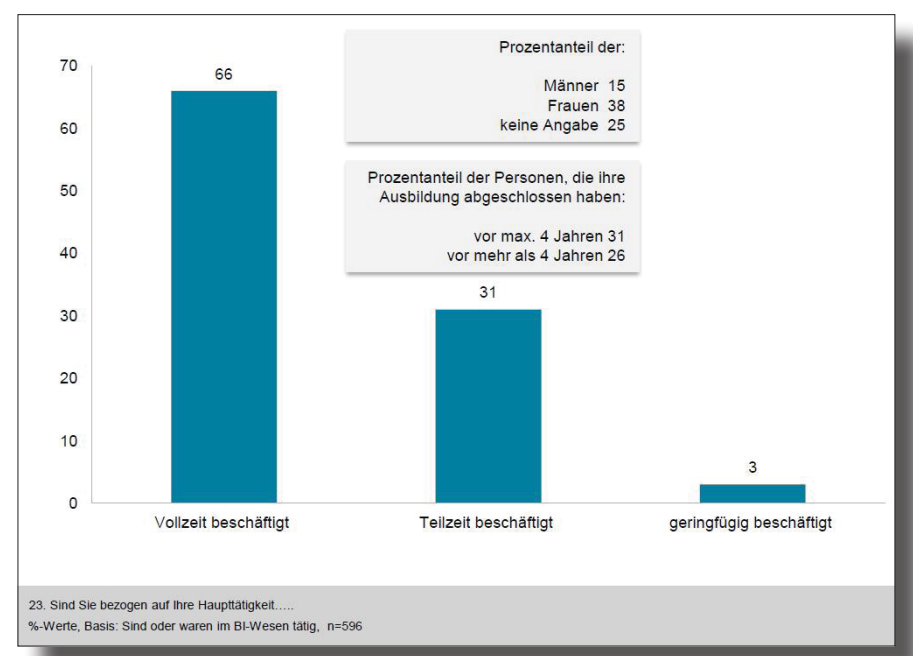

\subsubsection{Beschäftigungsstatus}

Mit 31\% Teilzeit arbeitenden KollegInnen liegt das Bibliothekswesen sogar etwas über den gesamtösterreichischen Durchschnitt von 29,1\% bei unselbständig Beschäftigten, die Tatsache, dass deutlich mehr Frauen als Männer diese Beschäftigungsform wählen, findet sich aber in allen Berufen. ${ }^{3}$

\subsubsection{Gründe für Teilzeitbeschäftigung}




Bei differenzierter Betrachtung des Themas zeigt sich aber, dass die Teilzeitbeschäftigung von etwa der Hälfte der KollegInnen keineswegs freiwillig gewählt ist.

\subsubsection{Ausmaß an Wochenstunden}

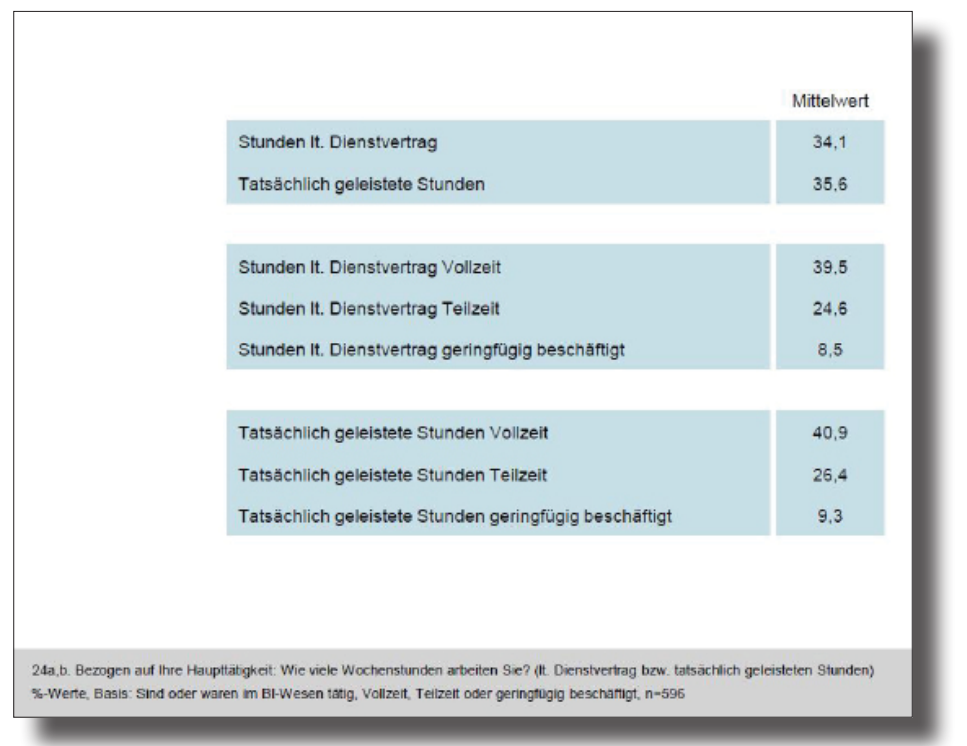

Betrachtet man unter diesem Aspekt die Diskrepanz zwischen den Stunden, die laut Dienstvertrag zu leisten sind und den Stunden, die regelmäßig tatsächlich geleistet werden - im Durchschnitt 1,8 Stunden pro Woche mehr bei Teilzeitbeschäftigten! -, zeigt sich sehr deutlich, dass einerseits das Leistungsvermögen von Dienstgebern offenbar häufig fehleingeschätzt wird und andererseits es ausreichend zu tun gäbe, um MitarbeiterInnen mehr Stunden zu beschäftigen. Darauf, dass dafür meist die finanziellen Mittel fehlen und der Handlungsspielraum bei derlei Entscheidungen auch für Bibliotheksleiterlnnen häufig sehr begrenzt ist, sei an dieser Stelle deutlich hingewiesen.

\subsubsection{Situation gegenüber Vollzeitbeschäftigten}

Aber die Ergebnisse zeigen, dass es über eine etwaige Erhöhung des Beschäftigungsausmaßes hinaus einfacher umzusetzende Maßnahmen gäbe, um Teilzeitkräfte verstärkt ins Team einzubinden und ihnen das Gefühl zu geben, auch als Teilzeitkraft eine vollwertige Mitarbeiterin zu sein. Denn von der Teilnahme an Weiterbildungsmöglichkeiten, Konferenzen und Workshops 
profitiert letztendlich auch die Dienstgeberin, der Aufwand einer Gleichstellung bei der Teilnahme an Betriebsveranstaltungen (Weihnachtsfeier, Betriebsausflug...) kann wohl mit erhöhter Motivation und Arbeitsleistung durch verstärktes Zugehörigkeitsgefühl zur Bibliothek aufgewogen werden und eine Berücksichtigung bei der Teilnahme an internen Besprechungen erhöht zwar den Planungsaufwand (und wird auch nicht in allen Fällen und immer möglich sein), stellt aber auf der anderen Seite die Einbeziehung der Teilzeitkräfte in den internen Informationsaustausch sicher.

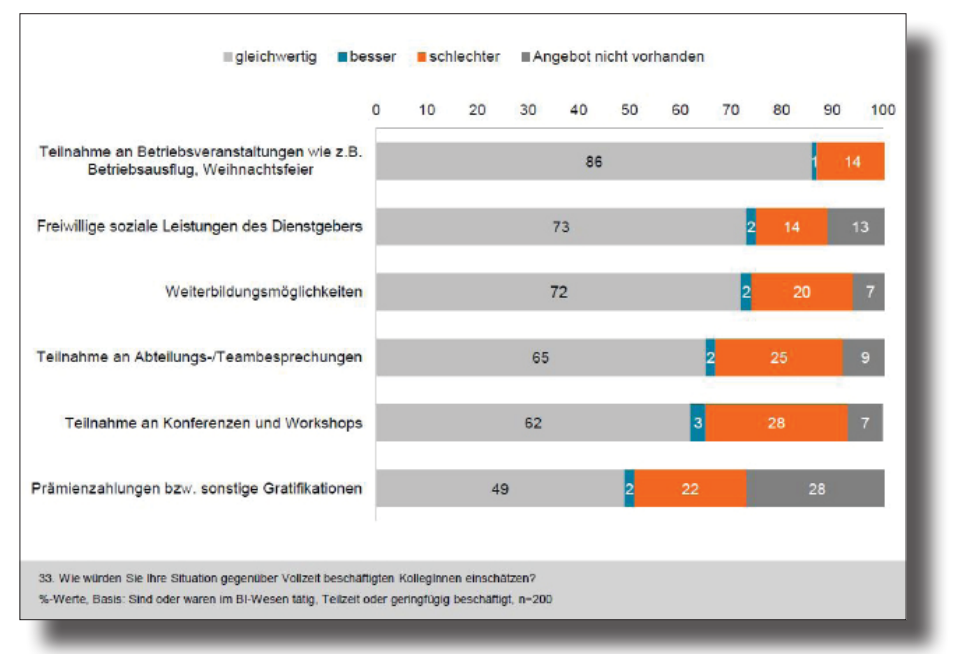

3.4.7. Zufriedenheit mit der Haupttätigkeit




Trotz der angesprochenen Probleme zeigen sich die KollegInnen weitestgehend zufrieden mit ihrer bibliothekarischen Tätigkeit. Als Hauptursachen für Unzufriedenheit werden Unterforderung, mangelnde Führungsqualität und schlechtes Betriebsklima sowie Überforderung durch Arbeitsüberlastung und zu hohe Erwartungshaltungen genannt, nur nachgereiht finden sich Bezahlung und prekäre/unsichere Arbeitsverhältnisse.

\subsubsection{Gehaltseinstufung in Bezug auf Ausbildung}

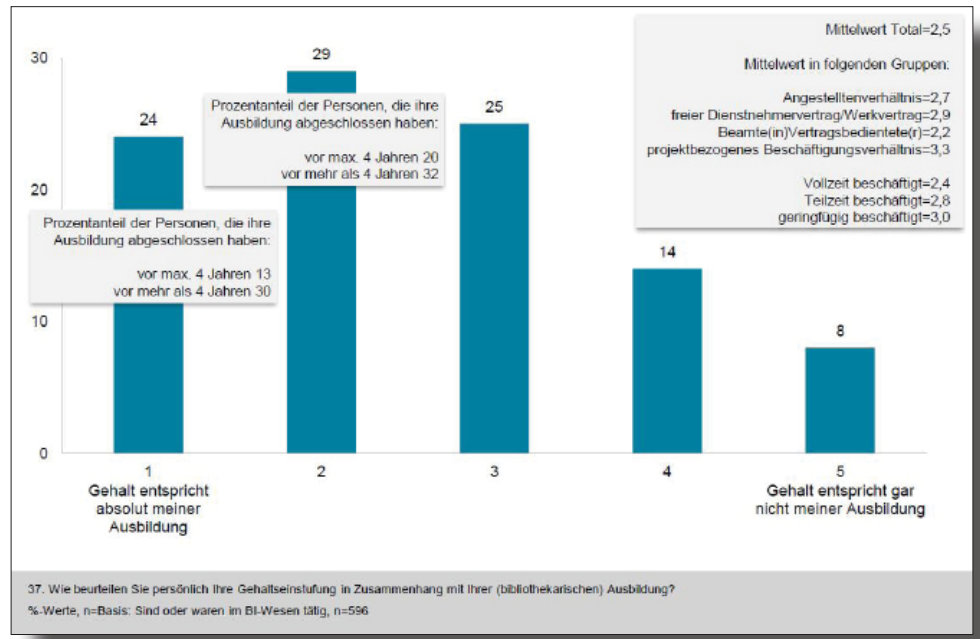

Dies ist insofern bemerkenswert, als fast die Hälfte der Befragten der Meinung sind, dass ihre Gehaltseinstufung mäßig bis gar nicht ihrer Ausbildung entspricht, wobei der Anteil der wenig bis gar nicht Zufriedenen steigt, je kürzer die Beschäftigungsdauer im Bibliothekswesen und je unsicherer das Beschäftigungsverhältnis ist.

\subsubsection{Sicherheit des Arbeitsplatzes}

Wenig überraschend ist das Ergebnis, dass das subjektive Unsicherheitsgefühl umso stärker ausgeprägt ist, je weniger Chancen aufWeiterbeschäftigung gesehen werden - besonders deutlich ausgeprägt ist es bei projektbezogenen Beschäftigungsverhältnissen. Auch allgemeine Sparmaßnahmen und die Abhängigkeit des Bibliothekswesens von staatlichen Budgetmitteln und damit politischen Verhältnissen werden als Unsicherheitsfaktoren angegeben. 


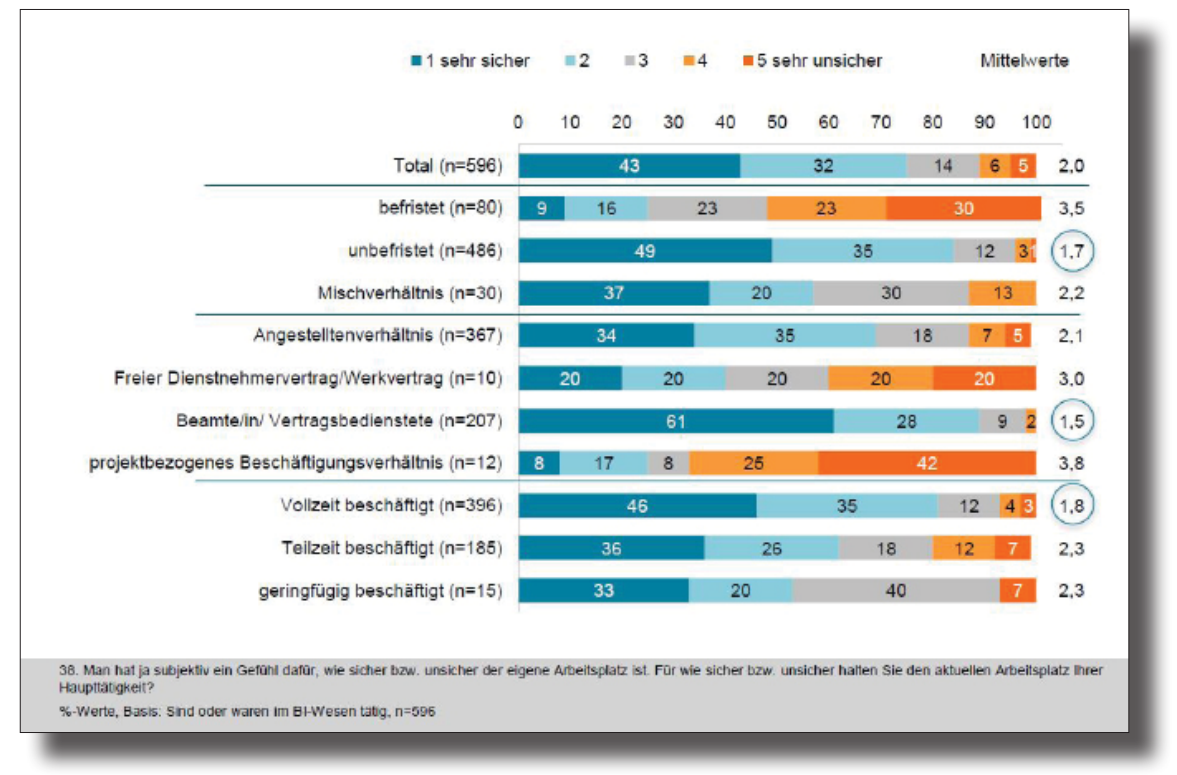

\subsection{Die berufliche Zukunft}

\subsubsection{Zukunftsszenario}

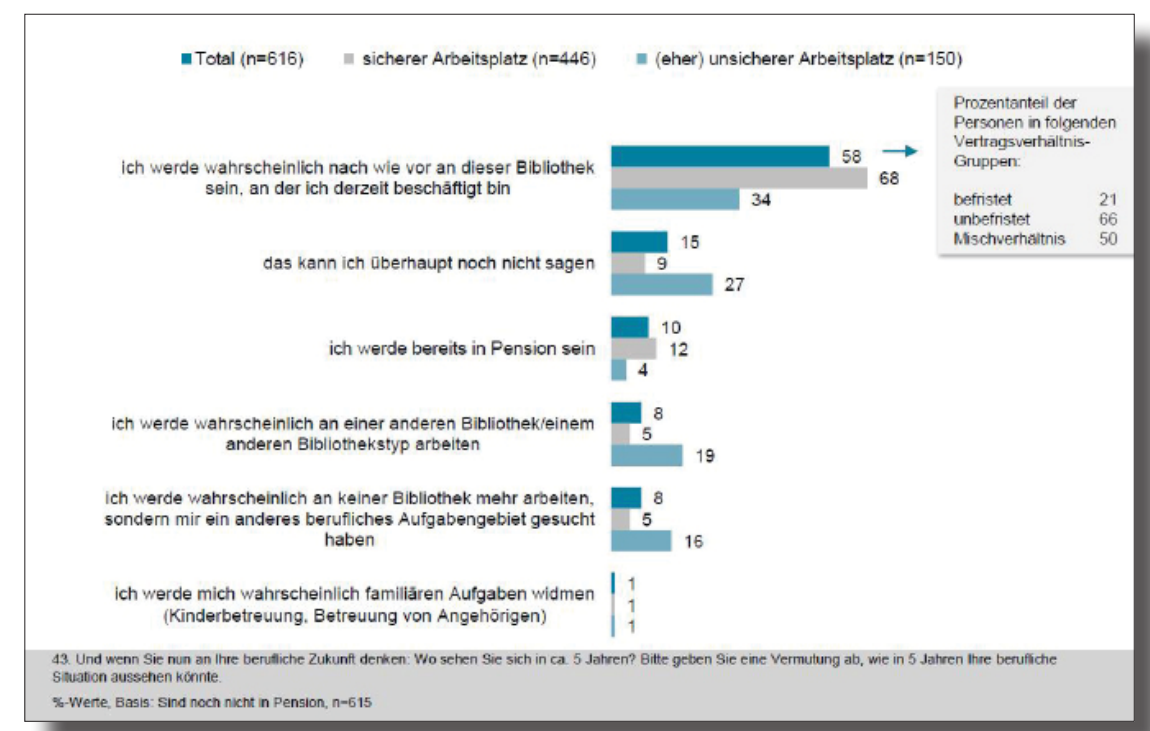


Auch hier zeigt sich, dass KollegInnen umso pessimistischer in die Zukunft blicken - sowohl was ihre Beschäftigung an derselben Einrichtung als auch ihre Beschäftigung im Bibliothekswesen allgemein betrifft -, je unsicherer sie ihre berufliche Situation empfinden. Diese Gruppe ist besonders anfällig sowohl für Überarbeitung, um durch besonders hohen Einsatz die Chancen auf eine Weiterbeschäftigung zu erhöhen, als auch für Geringleistung auf Grund „innerer Kündigung“, der kontinuierlichen Suche nach einem anderen, sichereren Job bzw. der Hemmung, sich zu sehr auf eine Tätigkeit/ein Team einzulassen und somit den Trennungsschmerz bei Vertragsende zu minimieren.

\subsubsection{Wunscharbeitszeit - Tatsächlich geleistete Arbeitszeit}

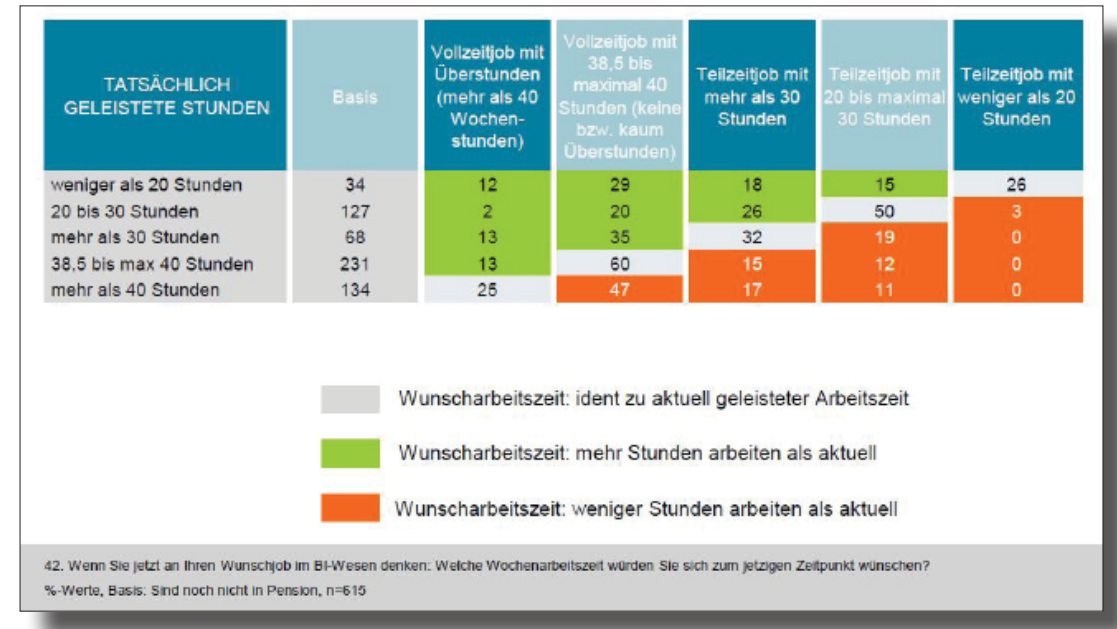

Es zeigt sich, dass Wunsch- und tatsächliche Arbeitszeit nicht nur bei Teilzeitbeschäftigten auseinanderklaffen. Auch wenn es wohl für viele KollegInnen Gründe innerhalb und außerhalb ihres Einflussbereiches gibt, warum keine Angleichung zwischen Wunsch und Wirklichkeit erfolgen kann, läge hier - auch mit Blick auf geleistete Überstunden - mittelfristig eine Möglichkeit, durch teilweise Neuverteilung von Arbeitsstunden und Aufgaben (sofern fachlich machbar) etwas mehr Ausgewogenheit herzustellen.

Weitere Veränderungswünsche an die berufliche Zukunft betreffen u.a.

- die Verbesserung der Gehaltssituation, insbesondere die Angleichung der Bezahlung an Tätigkeiten und dafür benötigte Qualifikationen, 
- verbesserte Aus- und Weiterbildung durch bedarfsorientiertere/zukunftsweisendere Inhalte und Verringerung der Ausbildungsplätze, um ein Überangebot an AbsolventInnen zu vermeiden,

- eine Erhöhung der Jobsicherheit,

- anspruchsvollere Tätigkeiten mit besseren Aufstiegschancen sowie mehr Eingebundenheit in Entscheidungen sowie

- bessere Führungskräfte bzw. besseres Betriebsklima mit mehr persönlicher Anerkennung und Wertschätzung.

\section{Zusammenfassung - erste Schlussfolgerungen}

Im Rahmen der abschließenden AG-Sitzung am 2.02.2018 befassten sich alle AG-Mitglieder nochmals intensiv mit den Ergebnissen, erstellten Problemskizzen, formulierten Ziele, welche Verbesserungen erreicht werden sollen und konkrete Vorschläge, um diese zu erreichen. Zusammenfassend lässt sich sagen: ${ }^{4}$

Grundsätzlich sind die Arbeitsverhältnisse im bibliothekarischen Bereich - auch im Vergleich mit den Beschäftigungsdaten der Statistik Austria (Teilzeitbeschäftigung, Medianeinkommen nach Bildungsabschluss...) - nicht so besorgniserregend, wie der Ursprung der Bottom-Up-Debatte es hätte vermuten lassen. Trotzdem gibt es, wie die Auswertungen und Kommentierungen zeigen, einige Punkte, auf die besonderes Augenmerk gelegt werden soll. Folgende Maßnahmen wurden von der AG empfohlen, um die Auseinandersetzung mit dem Thema zu vertiefen und Lösungen für Verbesserungen zumindest in einigen Bereichen zu finden:

- Bericht der zentralen Ergebnisse im Rahmen einer ubifo-Sitzung, um die dort versammelten BibliotheksleiterInnen für diese Themen zu sensibilisieren und möglichst die Akzeptanz und Unterstützung der Vorschläge zu erreichen. - Dies erfolgte durch Claudia Hausberger bei der ubifo-Sitzung am 7.6.2018, wo die präsentierten Ergebnisse durchaus auf Interesse stießen.

- Von der VÖB veranstalteter Ganztags-Workshop: eingeladen werden Bibliotheksleitungen (zumindest Uni-Bibliotheken, ggf. auch darüber hinaus, um mehrere Bibliothekstypen vertreten zu haben), Betriebsratsvertretungen der Bibliotheken und Ausbildungsverantwortliche - in mehreren Panels Diskussion der Ergebnisse und Verbesserungsvorschläge. Ziel ist es, am Ende des Tages für die Dinge, die betriebsintern einfach umzusetzen sind (verbesserte Integration von Teilzeitkräften, Betonung der Wichtigkeit von Jahresgesprächen 
und verbindliche Besprechung von Laufbahnperspektiven etc.), eine gemeinsame verbindliche Vereinbarung zu treffen und für die Punkte, die über die Rektorate, die Gewerkschaft (Kollektivvertrag...) etc. einzuhängen sind, gemeinsame Sichtweisen und abgestimmte Vorgehensweisen zu finden. - Ist noch offen, da sehr ressourcenintensiv (Zeit, Geld, Personal...).

- VÖB: Abhaltung einer Diskussionsveranstaltung/eines Workshops zu den Bereichen Berufsbild, Aus- und Weiterbildung, Laufbahnplanung, Gehalt vs. erforderliche Qualifikationen etc. für alle Interessierten, um auch als Berufsvereinigung die Wünsche und Bedürfnisse, Ansichten und Lösungsvorschläge der Mitglieder/ aller BibliothekarInnen im Austausch mit ExpertInnen zu erfahren. - Ist geplant im Rahmen des Österreichischen Bibliothekartags 2019 in Graz.

- VÖB: Schaffung einer Anlaufstelle (eine Person fix als Ansprechperson nominieren und entsprechenden Bereich im Webauftritt gestalten) v.a. für Neu- und WiedereinsteigerInnen: Infos zum Berufseinstieg, zu Aus- und Weiterbildung, zu Jobplattformen etc., Infos für die ULGs, Gestaltung einer „Infomappe“ (auch in Website-, Blogoder Wiki-Form) - Etablierung der VÖB als Anlaufstelle für Newcomer à Vortrag und Diskussion zum Berufseinstieg im Rahmen des ULG wurde 2018 erstmals durchgeführt, alle weiteren Maßnahmen sind in Planung für das VÖB-Präsidium 2019-2021.

- VÖB: Erstellung einer Konkordanz zwischen ausgeschriebenen Anforderungen und empfohlenen KV-Einstufungen mit dem Ziel, dass zumindest alle Unis einheitlich bewerten; ist nötig, da in den beispielhaft gelisteten Tätigkeiten die neuen Bereiche überhaupt nicht abgebildet sind. - Ist noch offen, da sehr ressourcenintensiv (Zeit, Personal...).

- Desiderat: da einige Ergebnisse (noch?) nicht besorgniserregend sind, aber doch auffallen - in 5 Jahren Wiederholung der Umfrage mit denselben Fragestellungen, um aus den Antworten erkennen zu können, was sich verbessert/was sich verschlechtert hat, welche Maßnahmen gegriffen haben, wo weitere Maßnahmen überlegt werden müssen?

Dass das Thema der zunehmenden atypischen Beschäftigungsverhältnisse mit einhergehender gesteigerter Gefahr der Prekarisierung nicht nur das österreichische Bibliothekswesen betrifft, zeigte sich beim 107. Deutschen Bibliothekartag 2018 in Berlin. Unter dem Titel „Qualifiziert, motiviert, befristet und in Teilzeit. Wie prekär ist das Bibliothekswesen?" lud der 
VDB-Regionalverband Berlin-Brandenburg zu einem zweistündigen Themenblock mit Podiums- und Publikumsdiskussion, bei welchem auch die Ergebnisse und Erkenntnisse der Umfrage der AG präsentiert werden konnten. Ein voll besetzter Saal und eine lebhafte Diskussion machten deutlich, dass die Probleme im Bibliothekswesen in Deutschland ähnlich gelagert sind, wenn auch die kollektivvertragliche Einstufung als nicht ganz so deutlich unterbezahlt erachtet wurde.

Alle Teilnehmerlnnen der AG Prekäre Arbeitsverhältnisse haben (selbstverständlich ehrenamtlich, wie bei allen Tätigkeiten im Rahmen der VÖB!) sehr viel Zeit und Energie investiert, um Ergebnisse zu liefern, die als valide Grundlage für weitere Maßnahmen dienen können. Nun bleibt zu hoffen, dass sich auch für die nächsten Schritte - die sukzessive Umsetzung der empfohlenen Maßnahmen, sofern sie in den Händen jedes/jeder Einzelnen liegen - genügend engagierte Kolleginnen und Kollegen finden. Bei Interesse bitte melden bei: ute.woedl@akwien.at

Mag. ${ }^{a}$ Ute Wödl, MSc Arbeiterkammer Wien, Bibliothek - Wissen - Information E-Mail: ute.woedl@akwien.at

1 https://personalwesen.univie.ac.at/fileadmin/user_upload/d_personalwesen/Jobs_Recruiting/Dokumente/Kollektivvertrag_Universitaeten. pdf (letzter Zugriff: 2.12.2018)

2 Die vollständige Präsentation der Ergebnisse ist hier zu finden: https:// opus4.kobv.de/opus4-bib-info/frontdoor/index/index/searchtype/collection/id/16673/start/0/rows/20/facetNumber_author_facet/all/author_facetfq/Weiner\%2C+Ute/docld/3258 (letzter Zugriff: 2.12.2018)

3 https://www.statistik.at/web_de/statistiken/menschen_und_gesellschaft/arbeitsmarkt/arbeitszeit/teilzeitarbeit_teilzeitquote/index.html (letzter Zugriff: 2.12.2018)

4 Eine umfassende Ausarbeitung an möglichen Handlungsfeldern und Empfehlungen findet sich im Abschlussprotokoll: https://www.univie. ac.at/voeb/kommissionen/ag-prekaere-arbeitsverhaeltnisse-im-bibliothekswesen/ (letzter Zugriff: 2.12.2018) 